\title{
Senecio madagascariensis Poir. (Asteraceae): uma nova causa de seneciose em bovinos no Sul do Rio Grande do Sul ${ }^{1}$
}

\author{
Adriana Lücke Stigger ${ }^{2}$, Pablo Estima-Silva² ${ }^{2}$ Leticia Fiss ${ }^{2}$, Ana Carolina B. Coelho ${ }^{2}$, Bianca \\ L. Santos ${ }^{2}$, Dale R. Gardner ${ }^{3}$, Clairton Marcolongo-Pereira ${ }^{4 *}$ e Ana Lucia Schild ${ }^{4 * *}$
}

\begin{abstract}
Stigger A.L., Estima-Silva P., Fiss L., Coelho A.C.B., Santos B.L., Gardner D.R., Marcolongo-Pereira C. \& Schild A.L. 2014. [Senecio madagascariensis Poir. (Asteraceae): a new cause of seneciosis in cattle in Southern Brazil.] Senecio madagascariensis Poir. (Asteraceae): uma nova causa de seneciose em bovinos no Sul do Rio Grande do Sul. Pesquisa Veterinária Brasileira 34(9):851-855. Laboratório Regional de Diagnóstico, Faculdade de Veterinária, Universidade Federal de Pelotas, Campus Universitário s/n, Pelotas, RS 96010900, Brazil. E-mail: alschild@terra.com.br

Two outbreaks of Senecio madagascariensis poisoning in cattle occurred in October 2013 in southern Brazil. Morbidity rate ranged were 3.2\% and 6.1\%, and lethality rate was $100 \%$. A third case of poisoning occurred on a property in which out of 54 cattle one died with clinical signs of intoxication. In all cases, the cattle were in areas highly infested by $S$. madagascariensis that was in bloom. The clinical signs were diarrhea, tenesmus, opisthotonus, and progressive weight loss. Death occurred between 10 and 15 days after the onset of clinical signs. At necropsy, lesions were edema in the mesentery, in the wall of the rumen and abomasum, and in the walls of the gall bladder; the liver was firm with marbled aspect. Histologically, the liver had proliferation of fibrous connective tissue, especially in portal areas, hepatomegalocytosis, and bile duct hyperplasia. The observation of large number of $S$. madagascariensis in several farms in the counties of Arroio Grande, Pedro Osório and Capão do Leão reinforces that this plant is in the process of adaptation and dissemination in this region and that other outbreaks may occur in coming years. The cases reported here apparently resulted from the consumption of the plant during the fall/winter of 2013, when it was in bloom. The quantification of alkaloids in $S$. madagascariensis revealed the presence of $500 \mu \mathrm{g} / \mathrm{g}$ and $4000 \mu \mathrm{g} / \mathrm{g}$ of pyrrolizidine alkaloids in the dry plant from two properties. It is believed that the large amount of the plant in the areas where the animals were and the amount of pyrrolizidine alkaloids found were factors that contributed to the occurrence of outbreaks.
\end{abstract}

INDEX TERMS: Poisonous plants, Senecio madagascariensis, Asteraceae, hepatotoxic plants, seneciosis, plant poisoning, cattle.

RESUMO.- Descrevem-se dois surtos de intoxicação por Senecio madagascariensis Poir. diagnosticados em bovinos em outubro de 2013 na região sul do Rio Grande do Sul. A

\footnotetext{
${ }^{1}$ Recebido em 6 de agosto de 2014.

Aceito para publicação em 28 de agosto de 2014.

${ }^{2}$ Curso de Pós-Graduação em Veterinária, Faculdade de Veterinária (FV), Universidade Federal de Pelotas (UFPel), Campus Universitário s/n, Pelotas, RS 96015-560, Brasil.

${ }^{3}$ USDA-ARS Poisonous Plant Research Laboratory, 1150 East 1400 North, Logan, Utah 84341, USA.

${ }^{4}$ Laboratório Regional de Diagnóstico, FV-UFPel. *Bolsista de Pós-Doutorado Júnior, CNPq (Proc.150246/2014-5). **Autor para correspondência: alschild@terra.com.br
}

morbidade foi de $3,2 \%$ e de $6,1 \%$ respectivamente e a letalidade foi de $100 \%$. Um terceiro caso da intoxicação ocorreu em uma propriedade na qual de 54 bovinos um morreu com sinais clínicos da intoxicação. Em todos os casos, os bovinos estavam em áreas altamente infestas por $S$. madagascariensis que se encontrava em floração. Os sinais clínicos caracterizaram-se por diarreia, tenesmo, opistótono e emagrecimento progressivo e a morte ocorreu entre 10 e 15 dias após o início dos sinais clínicos. Nas necropsias as lesões eram de edema do mesentério, das paredes do abomaso e do rúmen, e das paredes da vesícula biliar, além de fígado firme e com aspecto marmorizado. Histologicamente havia no fígado proliferação de tecido conjuntivo fibroso, 
principalmente nos espaços porta, megalocitose e hiperplasia de ductos biliares. A observação de grande quantidade de $S$. madagascariensis em várias propriedades nos municípios de Arroio Grande, Pedro Osório e Capão do Leão a partir do ano 2013 sugere que esta planta está em pleno processo de adaptação e disseminação nesta região e que outros surtos podem ocorrer nos próximos anos. Os surtos relatados aparentemente resultaram do consumo da planta durante o outono/inverno de 2013, quando a mesma estava já em floração. A quantificação dos alcaloides revelou a presença de $500 \mu \mathrm{g} / \mathrm{g}$ e $4000 \mu \mathrm{g} / \mathrm{g}$ de planta seca de alcaloides pirrolizidínicos em duas das três propriedades com casos de seneciose. Acredita-se que a grande quantidade de planta existente nas áreas onde os animais estavam e a quantidade de alcaloides presentes na mesma foram fatores que determinaram a ocorrência dos surtos.

TERMOS DE INDEXAÇÃO: Plantas tóxicas, Senecio madagascariensis, Asteraceae, plantas hepatotóxicas, seneciose, intoxicação por plantas, bovinos.

\section{INTRODUÇÃO}

A intoxicação por Senecio spp. tem sido amplamente estudada, especialmente na região Sul do Brasil devido a sua importância como causa de morte de bovinos nesta região (Barros et al. 1992, Karam et al. 2004, Grecco et al. 2010). Uma das espécies do gênero, Senecio madagascariensis, que é nativa da Ilha de Madagascar e África do Sul, é importante invasora, especialmente nas regiões de latitude similar à da origem da planta (Scott et al. 1998, Sindel et al. 2009) e foi introduzida de forma acidental em diversos países como Austrália (Parsons \& Cuthbertson 2001), Havaí (Roux et al. 2006), Argentina (Villalba \& Fernández 2005) e Uruguai (Cabrera \& Ré 1965, Cabrera 1963, Lombardo 1983). A planta foi identificada, também, no Rio Grande do Sul em 1995 (Matzenbacher \& Schneider 2008). Esta planta possui uma grande capacidade de dispersão apresentando boa adaptação ambiental e climática devido a sua característica hibernal (Matzenbacher \& Schneider 2008).

Na Austrália têm sido relatados sérios problemas de competição que $S$. madagascariensis está causando na agricultura e na pecuária, tanto em solos cultivados como em áreas com pouca cobertura vegetal ou campos nativos negligenciados (Sindel 1994). Além disso, devido ao seu conteúdo em alcaloides pirrolizidínicos, são mencionados sérios problemas na sanidade dos rebanhos (Sindel 1994). No Rio Grande do Sul a intoxicação por S. madagascariensis foi descrita em uma propriedade leiteira com mortalidade de 1,26\% (Cruz et al. 2010).

Os objetivos do presente trabalho foi relatar a ocorrência de intoxicação por S. madagascariensis em 2013 na região Sul do Rio Grande do Sul, descrever sua epidemiologia, bem como estabelecer diferenças e/ou similaridades com a intoxicação por outras espécies de Senecio.

\section{MATERIAL E MÉTODOS}

Duas propriedades com histórico de mortalidade de bovinos com sinais clínicos de diarreia, emagrecimento progressivo e tenesmo retal foram visitadas no mês de outubro de 2013 pelos técnicos do Laboratório Regional de Diagnóstico da Faculdade de Veterinária da Universidade Federal de Pelotas (LRD/UFPel). Uma terceira propriedade também foi visitada após a morte de um bovino com sinais semelhantes aos descritos acima. Foram investigados os dados epidemiológicos e os sinais clínicos apresentados pelos bovinos. Quatro bovinos afetados, das três propriedades, foram necropsiados e fragmentos de todos os órgãos, incluindo o sistema nervoso central, foram fixados em formalina a $10 \%$, processados rotineiramente e corados com hematoxilina e eosina (HE). As áreas onde os animais estavam foram percorridas para observação da presença de espécies de Senecio já que a suspeita da mortalidade era de intoxicação por plantas deste gênero em função dos sinais clínicos observados. Amostras de uma espécie de Senecio desconhecida para proprietários/veterinários e trabalhadores rurais de duas das três propriedades, foram colhidas para identificação no Departamento de Botânica do Instituto de Biologia da UFPel e posteriormente foram encaminhadas para o United State Department of Agriculture - Agriculture Research Service/ Poisonous Plant Research Laboratory (USDA-ARS/PPRL) para a quantificação de alcaloides pirrolizidínicos presentes.

\section{RESULTADOS}

As propriedades estavam localizadas nos municípios de Pedro Osório (Propriedade A longitude S 3153'32.1" e latitude W 52 $53^{\prime} 30.0^{\prime \prime}$ ), Arroio Grande (Propriedade B longitude S32 $05^{\prime} 27.9^{\prime \prime}$ e latitude W 52 $56^{\prime} 05.2^{\prime \prime}$ ) e Capão do Leão (Propriedade C longitude S $31^{\circ} 48^{\prime} 21.7^{\prime \prime}$ e latitude W 52 $\left.31^{\circ} 58.3^{\prime \prime}\right)$. Na Propriedade A um lote de 125 bovinos com 1,5 anos de idade estava em um potreiro de campo nativo e uma semana após ter sido trocado para outra área dois animais apresentaram diarreia escura, tenesmo e opistótono. Os bovinos foram transferidos para outro local e mais dois adoeceram. Todos os quatro bovinos morreram em período de 10-15 dias após o início dos sinais clínicos. Na propriedade B morreram seis bovinos de aproximadamente três anos de idade, de um total de 98 os quais apresentaram diarreia e emagrecimento progressivo. $\mathrm{Na}$ Propriedade $\mathrm{C}$ de 54 bovinos pastoreando em uma área de pastagem de Panicum spp. morreu um com sinais clínicos de diarreia, emagrecimento progressivo e sinais neurológicos de agressividade e incoordenação.

Na Propriedade A todos os potreiros onde os animais haviam estado apresentavam grande quantidade de $\mathrm{Se}$ -

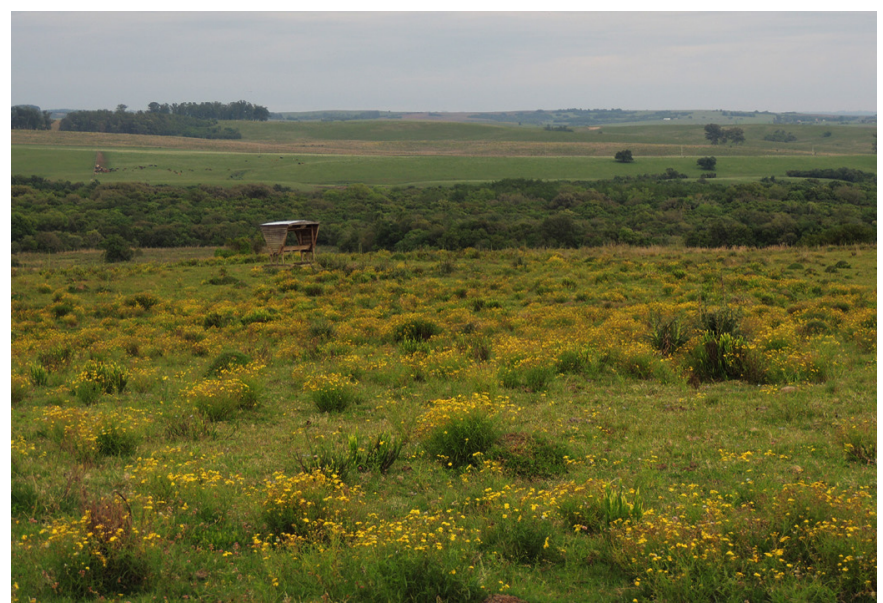

Fig.1. Área da propriedade A severamente invadida por Senecio madagascariensis. 


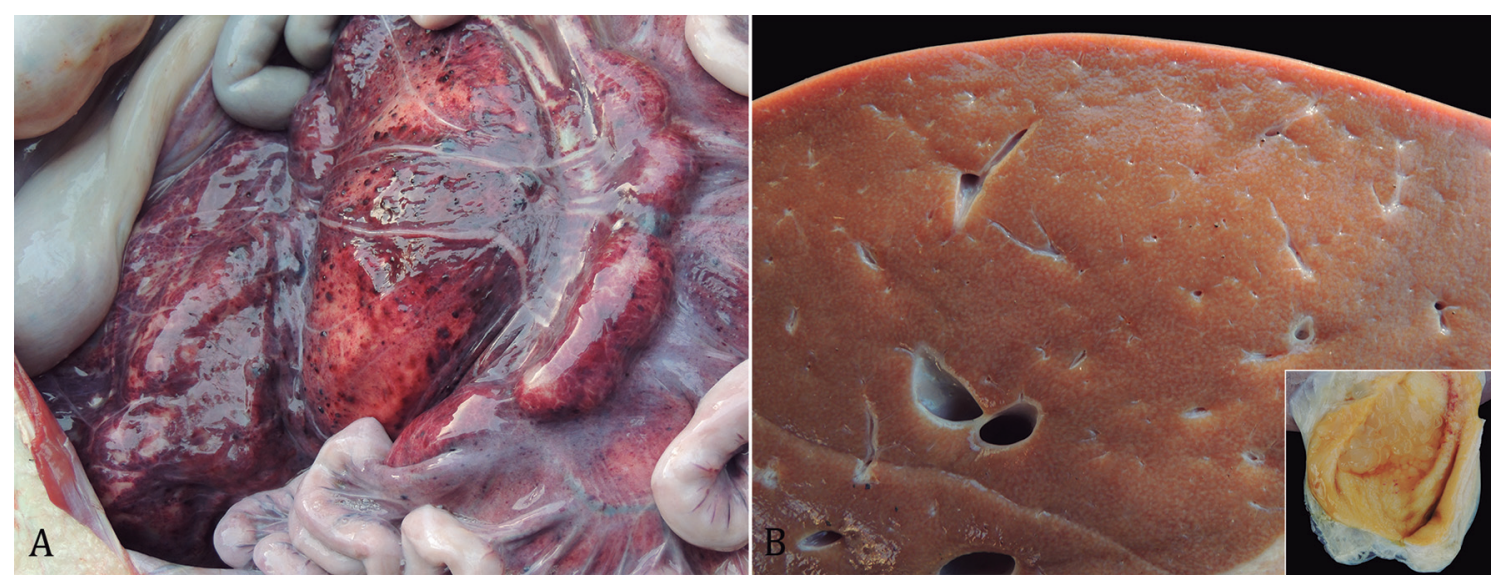

Fig.2. Intoxicação por Senecio madagascariensis em bovinos. (A) Edema hemorrágico no mesentério de um bovino necropsiado na Propriedade A. (B) Fígado de bovino da Propriedade C com aspecto marmorizado alternando pequenas áreas claras e áreas mais escuras. (Inset: Nodulações e edema da parede da vesícula biliar observados em bovino necropsiado na Propriedade B)

necio spp., posteriormente classificado como $S$. madagascariensis (Pel № 26323)(Fig.1). Na Propriedade B os bovinos foram transferidos de uma resteva de arroz do ano para uma pastagem de trevo com pouca disponibilidade de forragem onde permaneceram por 90 dias até o início dos sinais clínicos. Esta área estava, também, severamente invadida por S. madagascariensis. Na Propriedade C a planta (Pel № 26324) observada em grande quantidade estava misturada na pastagem de Panicum spp.

As amostras enviadas para quantificação dos alcaloides pirrolizidínicos no USDA-ARS/PPRL apresentaram níveis aproximados de $4000 \mu \mathrm{g} / \mathrm{g}$ de planta seca (Propriedade A) e $500 \mu \mathrm{g} / \mathrm{g}$ de planta seca (Propriedade C).

Macroscopicamente nos quatro bovinos necropsiados havia edema do mesentério que nos bovinos da Propriedade A era hemorrágico (Fig.2A). Havia, também, edema do abomaso e da parede do rúmen. 0 fígado, nos quatro bovinos, estava firme e esbranquiçado e ao corte apresentava um padrão marmorizado com áreas claras e outras mais escuras (Fig.2B). A vesícula biliar nos três casos estava aumentada de volume e apresentava edema da parede com petequias na mucosa e, em dois bovinos, havia hiperplasia polipoide (Fig.2B).

Histologicamente, no fígado havia proliferação de tecido conjuntivo fibroso, megalocitose e proliferação de ductos biliares nos quatro bovinos necropsiados (Fig.3) e estas lesões foram classificadas no Padrão de lesão 4, de acordo com Grecco et al. (2010).

\section{DISCUSSÃO}

No presente estudo o diagnóstico de intoxicação por Senecio madagascariensis foi realizado com base nos dados epidemiológicos, sinais clínicos, achados de necropsia, lesões histológicas e presença de grande quantidade da planta nas áreas onde os animais estavam.

Nas três propriedades onde ocorreram as mortes, de acordo com os proprietários, S. madagascariensis não era reconhecido como uma planta tóxica. Em um surto descrito em 2010, também no Rio Grande do Sul, os autores mencionaram o desconhecimento da planta por produtores e

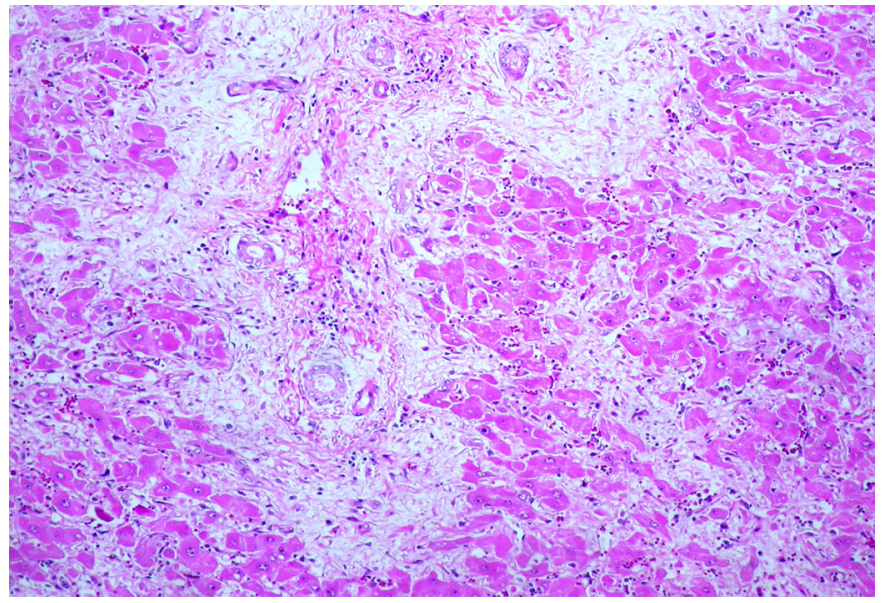

Fig.3. Aspecto histológico do fígado de bovino necropsiado na Propriedade B. Há proliferação de tecido conjuntivo fibroso periportal invadindo o parênquima hepático e hiperplasia biliar.

veterinários que afirmavam que não havia Senecio spp. no campo e em consequência disso a planta não foi controlada e ocorreu o surto (Cruz et al. 2010).

Apesar de S. madagascariensis ter sido observado a partir do ano 2013 não é possível precisar quando iniciou o processo de invasão e adaptação na região. 0 fato de produtores e veterinários desconhecerem a planta sugere que o aparecimento nestas áreas de campo foi recente e os bovinos também não haviam tido contato anterior com a mesma. Na Austrália foi mencionado que o desconhecimento da planta pelos animais pode facilitar a ocorrência da intoxicação quando os mesmos são transportados de áreas livres para áreas infestadas (Sindel \& Coleman 2012). Este fato poderia justificar pelo menos um surto descrito neste trabalho, no qual os animais foram transferidos de uma resteva de arroz nova para uma área invadida pela planta e não tinham tido contato anterior com $S$. madagascariensis ou outras espécies do gênero.

A observação de grande quantidade de $S$. madagascariensis em várias propriedades nos municípios de Arroio Grande, Pedro Osório e Capão do Leão a partir do ano 2013 sugere que esta planta está em pleno processo de adapta- 
ção e disseminação nesta região e que outros surtos podem ocorrer nos próximos anos. Tem sido mencionado que a dispersão das sementes pode ocorrer pelo vento (Sindel \& Coleman 2012) e esta provavelmente foi a forma de disseminação da planta nesta região.

A quantidade de alcaloides pirrolizidínicos presentes nas amostras analisadas justifica a ocorrência do surto na Propriedade A com uma morbidade de 3,2\%. 0 risco de ocorrência da intoxicação foi mencionado em bovinos pastoreando em áreas altamente infestadas com S. madagascariensis no Havaí, as quais continham entre 220 e 2000 $\mu \mathrm{g} / \mathrm{g}$ de planta seca de alcaloides pirrolizidínicos (Gardner et al. 2006).

Na Propriedade B a morbidade chegou a 6,12\% e isto sugere que provavelmente, nesta área a quantidade de alcaloides pirrolizidínicos nas plantas era semelhante. Em amostras provenientes do município de Eldorado do Sul no Rio Grande do Sul, analisadas durante as quatro estações do ano, houve variação na quantidade de alcaloides de $735-3490 \mu \mathrm{g} / \mathrm{g}$ de planta seca, sendo as maiores quantidades observadas na primavera e nas flores (Karam et al. 2011). Deve ser mencionado que $S$. madagascariensis nas três propriedades onde os surtos descritos ocorreram estava em floração desde o início do mês de agosto. Os bovinos na Propriedade A eram criados na área e na Propriedade B estavam no potreiro há pelo menos 3 meses, tempo suficiente para o desenvolvimento das lesões de seneciose.

Apesar da baixa concentração de alcaloides quando comparado ao conteúdo destas substâncias em uma amostra de $S$. brasiliensis, proveniente do município de Herval, onde foi diagnosticado um surto no ano anterior (Schild et al. 2013), que continha $35000 \mu \mathrm{g} / \mathrm{g}$ de planta seca, a grande quantidade de $S$. madagascariensis e a pouca disponibilidade de forragem foram fatores que contribuíram para a ocorrência dos surtos. Em trabalhos anteriores foram en-

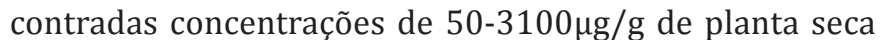
de diferentes alcaloides em diversas espécies de Senecio, que mostraram-se tóxicos para frangos e bovinos, experimentalmente (Méndez et al. 1989). Estas concentrações são inferiores à encontrada em $S$. madagascariensis da Propriedade A. Por outro lado são superiores às concentrações encontradas na planta da Propriedade C, no entanto deve se considerar a grande quantidade da planta existente na área onde o bovino desta propriedade foi intoxicado.

Não foram observadas outras espécies de Senecio nas três propriedades em que ocorreram os surtos descritos no presente trabalho, o que demonstra que nestes casos não há a possibilidade da intoxicação ter sido causada por outras espécies do gênero, que muitas vezes, se há condições climáticas favoráveis, podem estar disponíveis aos animais durante o ano todo (Karam et al. 2002).

Com relação às lesões observadas nos bovinos necropsiados a presença de edema hemorrágico em um bovino e o padrão marmorizado do fígado são similares às lesões da intoxicação por outras espécies de Senecio, especialmente S. brasiliensis. Em outro relato da intoxicação o padrão de lesão hepática descrito caracterizava-se pela presença de nódulos de 2-6mm (Cruz et al. 2010), que não foram observados nos bovinos no presente trabalho. Uma lesão até então não descrita foi o edema acentuado da parede do rúmen observado nos bovinos do surto da Propriedade A. Certamente o padrão de lesão hepática está muito mais relacionado a quantidade e tipo de alcaloides presentes e à quantidade e ao tempo de ingestão da planta do que à espécie de Senecio envolvida.

Conclui-se pelos resultados deste trabalho que $S$. $m a$ dagascariensis é um possível risco de intoxicação em áreas onde está presente devido ao seu grande potencial de dispersão e adaptação, tanto em pastagens como em campos nativos e poderá ser um grave problema nos próximos anos para a pecuária da região. São necessárias medidas de controle imediatas para minimizar prejuízos decorrentes desta intoxicação, especialmente, por tratar-se de uma espécie ainda desconhecida como tóxica tanto para produtores como para veterinários.

Agradecimentos.- Trabalho financiado pelo INCT/CNPq (Proc.573534/ 2008-0).

\section{REFERÊNCIAS}

Barros C.S.L., Driemeier D., Pilati C., Barros S.S. \& Castilhos L.M.L. 1992. Senecio spp. poisoning in cattle in Southern Brazil. Vet. Hum. Toxicol. 34(3):241-246.

Cabrera A.L. 1963. Flora de la Provincia de Buenos Aires: Compuestas. Coleccion Científica del INTA, Buenos Aires, 4(6). 433p.

Cabrera A.L. \& Ré R.R. 1965. Sobre un Senecio adventicio en la Provincia de Buenos Aires. Revta Facultad de Agronomía de la Universidad Nacional de La Plata, La Plata, 41(1):43-50.

Cruz C.E.F., Karam F.C., Dalto A.C., Pavarini S.P., Bandarra P.M. \& Driemeier D. 2010. Fireweed (Senecio madagascariensis) poisoning in cattle. Pesq. Vet. Bras. 30:10-12.

Gardner D.R., Throne M.S., Molyneaux R.J., Foster J.A. \& Seawright A.A. 2006. Pyrrolizidine alkaloids in Senecio madagascariensis from Australia and Hawaii and assessment of possible livestock poisoning. Biochem. Syst. Ecol. 34:736-744.

Grecco F.B., Schild A.L., Estima-Silva P., Marcolongo-Pereira C., Soares M.P. \& Sallis E.S.V. 2010. Aspectos epidemiológicos e padrões de lesões hepáticas em 35 surtos de intoxicação por Senecio spp. em bovinos no sul do Rio Grande do Sul. Pesq. Vet. Bras. 30(5):389-397.

Karam F.S.C., Méndez M.C., Jarenkow J.A. \& Riet-Correa F. 2002. Fenologia de quatro espécies tóxicas de Senecio (Asteraceae) na região Sul do Rio Grande do Sul. Pesq. Vet. Bras. 22(1):33-39.

Karam F.S.C., Soares M.P., Haraguchi M., Riet-Correa F., Méndez M.C. \& Jarenkow J.A. 2004. Aspectos epidemiológicos da seneciose na região sul do Rio Grande do Sul. Pesq. Vet. Bras. 24:191-198.

Karam F.S.C., Haraguchi M. \& Gardner D. 2011. Seasonal variation in pyrrolizidine alkaloid concentration and plant development in Senecio madagascariensis Poir. (Asteraceae) in Brazil, p.179-185. In: Riet-Correa F., Pfister J., Schild A.L. \& Wierenga T. (Eds), Poisoning by Plants, Mycotoxins and related Toxins. CAB International, Wallingford, UK.

Lombardo A. 1983. Flora Montevidensis. Intendencia Municipal de Montevideo, Montevideo, 2:274-288.

Matzenbacher N.I. \& Schneider A.A. 2008. Nota sobre a presença de uma espécie adventícia de Senecio (Asteraceae) no Rio Grande do Sul, Brasil. Revta Bras. Biociênc. 6:111-115.

Parsons W.T. \& Cuthbertson E.G. 2001. Fireweed, Senecio madagascariensis Poir., p.304-305. In: Ibid. (Eds), Noxious Weeds of Australia. $2^{\text {nd }}$ ed. CSIRO Publishing, Collingwood, Victoria, Australia.

Roux J., Wieczorek A.M., Ramadan M.M. \& Tran C.T. 2006. Resolving the native provenance of invasive fireweed (Senecio madagascariensis Poir.) in 
the Hawaiian Islands as inferred from phylogenetic analysis. Div. Distrib. 12:694-702.

Schild A.L., Marcolongo-Pereira C., Fiss L., Santos B.L., Coelho A.C.B. \& Sallis E.S.V. 2013. Doenças diagnosticadas pelo Laboratório Regional de Diagnóstico no ano 2012. Bolm Lab. Reg. Diagn. 35, Pelotas. 63p.

Sindel B.M. 1994. Impact, ecology and control of the weed Senecio madagascariensis in Australia, p.339-349. In: Caligari P.D.S. \& Hind D.J.N (Eds), Proceedings of the International Compositae Conference. Vol.2. The Royal Botanic Gardens, Kew.

Sindel B., Jhorar O., Reeve I., Thompson L., Moss J. \& Coleman M. 2009.
Weed Detection on Farms: a guide for landholders. University of New England, Armidale.

Sindel B. \& Coleman M. 2012. Fireweed: a best practice management guide for Australian landholders. University of New England. 33.p. ISBN 9781-921597-37-4.

Scott L.J., Congdon C. \& Playford J. 1998. Molecular evidence that fireweed (Senecio madagascariensis, Asteraceae) is of South African origin. Plant Syst. Evol. 213:251-257.

Villalba J. \& Fernández G. 2005. Otra flor amarilla peligrosa: Senecio madagascariensis. Tambo 150:46-48. 